Pulsar Astronomy — 2000 and Beyond

ASP Conference Series, Vol. $3 \times 10^{8}, 1999$

M. Kramer, N. Wex, and R. Wielebinski, eds.

\title{
A Search for Sub-millisecond Pulsations in Unidentified FIRST and NVSS Radio Sources
}

\author{
Fronefield Crawford, Victoria M. Kaspi \\ Center for Space Research, MIT, Cambridge, MA 02139, USA \\ Jon F. Bell \\ ATNF, CSIRO, P.O. Box 76, Epping, NSW, Australia
}

\begin{abstract}
We have searched 92 unidentified sources from the FIRST and NVSS radio catalogs for pulsations at $610 \mathrm{MHz}$. The selected sources are bright, have no identifications, are unresolved and have significant linear polarization. Our search was sensitive to sub-millisecond pulsations from pulsars with a large range of dispersion measures. We have detected no radio pulsations from these sources and conclude that they are unlikely to be a population of previously undetected pulsars.
\end{abstract}

The FIRST and NVSS surveys are recent $1400 \mathrm{MHz}$ VLA radio surveys of the Northern sky. In the published FIRST catalog (White et al. 1997), the positions and fluxes of $\sim 1.4 \times 10^{5}$ discrete radio sources are complete to $\sim 1 \mathrm{mJy}$. The NVSS survey (Condon et al. 1998) catalogs more than $1.8 \times 10^{6}$ sources complete to $\sim 2.5 \mathrm{mJy}$ and preserves polarization information. Several large-scale pulsar surveys have been conducted at high Galactic latitudes (see Camilo 1997 for a review) which were sensitive to sub-millisecond pulsars with only a small range of dispersion measures $(\mathrm{DM} \lesssim 10 \mathrm{pc} \mathrm{cm}-3$ ). A targeted search of unidentified FIRST and NVSS sources for sub-millisecond pulsars with a wide range of DMs is feasible using small frequency channel widths and a fast sampling rate.

We have searched for radio pulsations in 92 bright, point-like unidentified sources from the FIRST and NVSS surveys which are more than $5 \%$ linearly polarized at $1400 \mathrm{MHz}$. Although there is no abrupt cutoff separating the pulsar population from the extragalactic radio source population, a polarization threshold of $5 \%$ excludes most $(\sim 90 \%)$ of the identified non-pulsar population while retaining the majority $(\sim 90 \%)$ of the identified pulsar population in the NVSS survey (Han \& Tian 1999). Each source was observed at a center frequency of $610 \mathrm{MHz}$ in two orthogonal linear polarizations for $420 \mathrm{sec}$ with the Lovell 76meter radio telescope. A bandwidth of $1 \mathrm{MHz}$ was split into 32 channels with signals from each channel recorded as a continuous 1-bit digitized time series sampled at $50 \mu \mathrm{s}$. We did not detect any pulsations from the target sources.

We argue that our non-detections imply that the sources are unlikely to be pulsars. The selected sources were bright, with the weakest source having a $1400 \mathrm{MHz}$ flux density of $15 \mathrm{mJy}$. Assuming a typical pulsar spectral index of $\alpha=1.6$ (Lorimer et al. 1995), this source would have a flux density $58 \mathrm{mJy}$ at $600 \mathrm{MHz}$. This is significantly greater than our sensitivity limit for the expected 


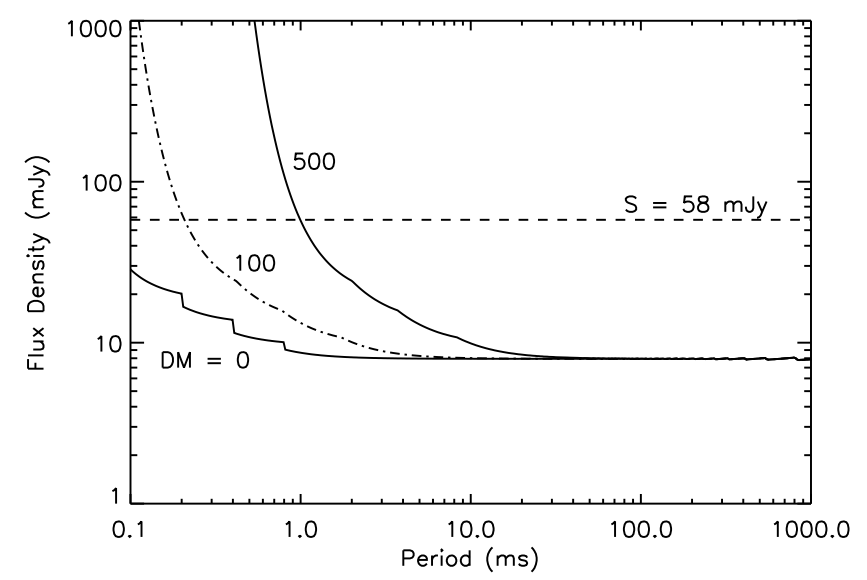

Figure 1. Pulsar sensitivity curves for DMs of 0, 100, and 500 pc $\mathrm{cm}^{-3}$, assuming a pulsed duty cycle of $5 \%$. The dashed horizontal line at $58 \mathrm{mJy}$ is the flux density of our weakest source at $600 \mathrm{MHz}$, assuming a spectral index of $\alpha=1.6$. All of our sources are expected to have $\mathrm{DM}<100 \mathrm{pc} \mathrm{cm}^{-3}$ (indicated by the dashed-dotted line).

range of pulse periods and DMs (see Fig. 1). All of our sources, therefore, were bright enough in the absence of scintillation to be detectable.

Dispersion smearing is not a factor preventing detection, since all but two of these sources have high Galactic latitudes $\left(|b|>5^{\circ}\right)$ and are expected to have $\mathrm{DM}<100 \mathrm{pc} \mathrm{cm}{ }^{-3}$ regardless of distance. This is well within our range of sensitivity to sub-millisecond pulsations ( $\mathrm{DM} \lesssim 500 \mathrm{pc} \mathrm{cm}{ }^{-3}$, Fig. 1$)$. Interstellar scattering, estimated from the Taylor \& Cordes (1993) model of the Galactic electron distribution, is expected to be negligible.

Scintillation could be preventing the detection of some of the sources if they are pulsars closer than $\sim 2 \mathrm{kpc}$. This distance range is reasonable: if the sources were pulsars at $2 \mathrm{kpc}$, their $400 \mathrm{MHz}$ luminosities would all be at the upper end of the pulsar luminosity distribution $\left(L_{400}>450 \mathrm{mJy} \mathrm{kpc}^{2}\right)$. However, it is unlikely that all of our sources would be suppressed in this way, so scintillation cannot account for the non-detection of most of our sources. We conclude that the question of the nature of these sources remains open. We have submitted an article to the Astronomical Journal which details the results presented here.

\section{References}

Camilo, F. 1997, in High Sensitivity Radio Astronomy, N. Jackson \& R. J. Davis, eds., 14

Condon, J. J. et al. 1998, AJ, 115, 1693

Han, J. L. \& Tian, W. W. 1999, A\&A, 136, 571

Lorimer, D., Yates, J., Lyne, A., \& Gould, D. 1995, MNRAS, 273, 411

Taylor, J. H. \& Cordes, J. M. 1993, ApJ, 411, 674

White, R. L., Becker, R. H., Helfand, D. J., \& Gregg, M. D. 1997, ApJ, 475, 479 